\title{
Tipificación y uso de los recursos de información en las organizaciones manufactureras
}

\author{
Tipification of the information resources used by manufacturing organizations
}

\author{
Celso Martínez Musiño \\ El Colegio de México, México D. F., Méjico \\ celsommm@yahoo.com.mx
}

\begin{abstract}
Resumen
Se analizan las investigaciones de tipo empírico sobre el uso de la información en las organizaciones manufactureras; y se identifican y tipifican los recursos de información utilizados por ese tipo de organizaciones. Para la tabulación de resultados de las búsquedas bibliográficas se ha utilizado la tipificación de recursos de información documental de las Reglas de Catalogación Angloamericanas. Se localizaron y analizaron siete investigaciones en las cuales se menciona el uso de información. Se ha identificado que las organizaciones manufactureras usan las monografías, los archivos de computadora y recursos digitales como recursos de información. A estos recursos se les han sumado las personas como fuente generación e intercambio de información.
\end{abstract}

Palabras clave: Información en las organizaciones. Administración de información. Gestión de información. Organizaciones manufactureras.

\section{Introducción}

Las organizaciones manufactureras se encuentran inmersas en una economía global, lo cual se traduce en mayor competencia por el mercado, y su permanencia en éste es cada vez más difícil. Uno de los principales argumentos para elaborar esta investigación es porque hay escasos estudios al respecto. Otro argumento que sustenta el interés por este estudio es que se asume que en toda organización se genera información, tanto de manera individual, como colectiva; las organizaciones manufactureras no son ajenas a este fenómeno. La investigación forma parte del desarrollo de la tesis doctoral Estudio de los flujos de información en las organizaciones bajo tres enfoques: administrativo, organizacional y ciencias de la información.

\begin{abstract}
A state of the question is provided on use information in manufacturer organizations with the aim of identifying and typifying the information resources used for those organizations. The information resources were classified according to the typification proposed in Anglo-American Cataloguing Rules. Seven research projects were identified providing information on the topic. Manufacturer organizations use monographies, computer files and digital resources as information resources. To those resources, studied organizations mentioned people as another key information resource.
\end{abstract}

Keywords: Information in organizations. Information management. Manufacturing organizations.

En investigaciones previas se encontró que: a) las organizaciones, independientemente de su tipología o tamaño, dan valor a la información y se apoyan en algunas disciplinas como la administración de información y la administración de recursos informativo; b) la información puede utilizarse como una herramienta para la consecución de los objetivos organizacionales mediante un tratamiento adecuado en los procesos de compilación, organización y uso de la información; c) la información no solamente ayuda a la toma de decisiones colectivas, mediante una administración y comunicación efectiva, sino que puede ser un detonante para convertirla en un activo y generar conocimientos colectivos $u$ organizacionales (Martínez Musiño, 2010, p. 18).

Las organizaciones en general, y las manufactureras en particular, no son ajenas al impacto 
que tiene, o puede tener, la identificación, la organización y el uso de la información como primera etapa para identificar sus flujos. Este tipo de organizaciones se enmarca en un contexto de la sociedad de la información, en la cual, además de enfrentarse a una economía y sociedad globalizadaa, tiene el reto de encontrar el valor de la información generada tanto de manera interna como externa. El conocimiento de ese valor puede ofrecer ventajas competitivas, al conocer las especificaciones propias de sus productos, las ventajas o desventajas respecto a los productos de la competencia, la opinión de los clientes respecto a sus productos y los de la competencia, a las posibilidades de diversificar sus márgenes de operaciones, o al seguimiento en el desarrollo de la adquisición de materias primas, por mencionar algunas ventajas.

Los objetivos de la investigación han sido los siguientes: a) buscar, identificar y analizar los documentos que refieran el uso de la información en las organizaciones manufactureras; b) identificar y tipificar las fuentes de información, e investigar el uso de la información de las organizaciones manufactureras a partir de una perspectiva de la ciencia de la información. Para cumplir con estos objetivos, la investigación se estructura de la siguiente manera: 1) la administración de información en las organizaciones: aspectos teóricos; 2) identificación y tipificación de la información en las organizaciones manufactureras; 3) método; 4) discusión; y, 5) conclusiones.

\section{La administración de información en las organizaciones: aspectos teóricos}

Esta investigación se focaliza en la Administración de Información (Al) y la Administración de Recursos de Información (ARI), razón por la cual es necesario describir sus antecedentes y definiciones. La Al y la ARI se relacionan con las funciones y tipos de recursos existentes en las organizaciones. En los años veinte se focalizaba en la administración financiera (dinero y capital); en la década siguiente la administración se centraba en las personas. En los años cuarenta, las funciones administrativas atendían principalmente los materiales, el equipo y las propiedades (terrenos y construcciones). Sin embargo, no es sino hasta la década de los setenta que la administración apuntaba hacia la energía y la información, y en la década siguiente, los ochenta, se centraba en el conocimiento (Figura 1).

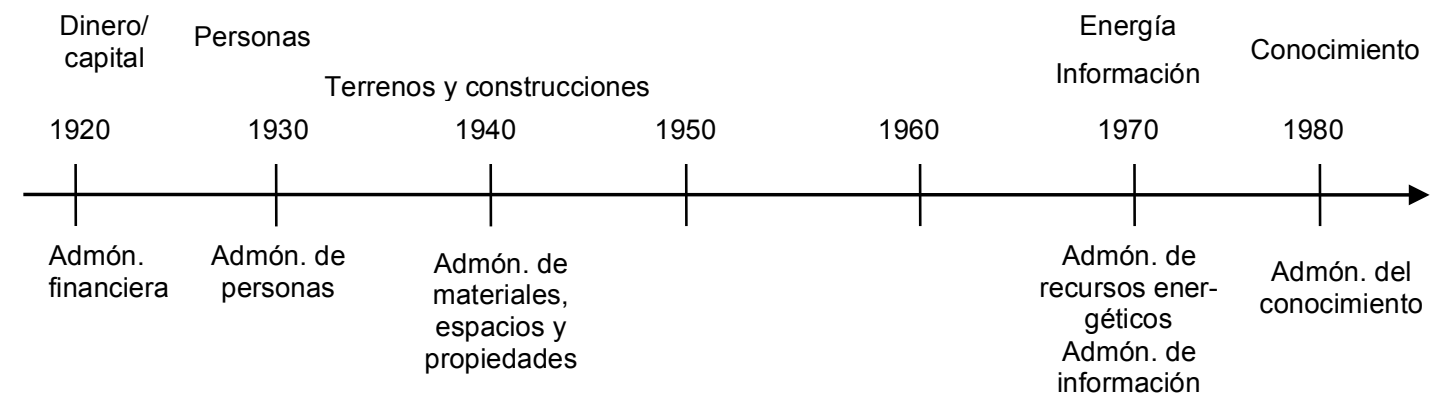

Figura 1. Evolución administración de recursos - recursos versus funciones (elaboración propia con datos de Burk \& Horton (1998)).

\begin{abstract}
¿Porqué investigar la información en las organizaciones manufactureras a partir de la Al y la ARI? Por un lado, Peter F. Drucker (2003, p. 49) vaticinaba que la información sería uno de los activos ${ }^{1}$ más importantes dentro y fuera de las organizaciones; mientras que, Elizabeth Orna, menciona la necesitad de establecer políticas prácticas de información con las cuales uno de sus beneficios favorecería la innovación exitosa, reduciendo la incertidumbre y los riesgos (Orna, 1990, p. 21). La autora señala, además, que uno de sus objetivos es conocer, cómo la información fluye a través de las organizaciones (Orna, 1990, p. 19).
\end{abstract}

La Al, según Archaira y Pani (2008, p. 145), requiere de ciertos elementos básicos, como el acceso, evaluación, administración, organización, filtrado y distribución de la información, convirtiéndose en información útil para el usuario final. Por su parte, Kirk (1999) indica que la Al es practicada por los individuos en las organizaciones. Por otro lado, Almeida y Escrivão (2006) focalizan más en los procesos de la Al, los cuales abrevian en varias etapas: en la determinación de necesidades de información y en la obtención, procesamiento, distribución y utilización de esa información. Respecto a la última etapa, los autores exteriorizan que, después de 
utilizada la información, hay una nueva solicitud de la misma, reiniciando un nuevo proceso de Al. Lo rescatable de la propuesta de Almeida y Escrivão, es la descripción del proceso en el cual se identifican las necesidades información, puesto que se puntualiza en la comprensión de las fuentes y los tipos de información, los cuales son obligatorios para un buen funcionamiento de las organizaciones.

El estudio de la información en las organizaciones, se puede realizar, además, a partir de ciertos contextos. Por ejemplo, Liao y Tang (2004), señalan que el estudio de la Al puede darse a partir de elementos tales como los datos, la información y el conocimiento. Los autores continúan, e indican que, en la dupla datoinformación, hay un proceso de integración, mientras que en la bina informaciónconocimiento hay un proceso de modelado y representación. Por un lado, la integración permite que las unidades de una organización perciban la importancia de conjuntar los datos para dar un sentido orientado a la obtención de metas organizacionales. Por otra parte, el modelado y representación, producto de integración de información, incide en la creación de capital humano mediante el conocimiento adquirido por los elementos de una organización.

Respecto a las organizaciones manufactureras, Forest Woody Horton, al mencionar la situación problemática del aumento de archivos de información sin organizar y la falta de valoración, tanto para la propia industria, como para sus competidores (Horton, 1993, p. 4), invitaba a aplicar la ARI. En el mismo sentido, con la premisa de comenzar a realizar inventarios de los recursos informativos en la industrias, Herrero y Torres (1993, 43-68) identificaron, tanto fuentes de información global como información sectorial para el uso de la industria española. La identificación de las fuentes de recursos de información, y su tipología, es uno de los primeros pasos para identificar los flujos en las organizaciones manufactureras (2).

En otro contexto de la Al, Bergeron (1997) contribuye, mediante enfoques cualitativos, al estudio de la práctica informativa utilizando la administración de recursos de información en las organizaciones para conocer quiénes y cómo los utilizan. Para esto, el autor, escogió, y categorizó, los siguientes sujetos de estudio: los directivos de distintas organizaciones de los sectores servicios e industrial.

\section{Identificación y tipificación de la información en las organizaciones manufactureras}

Después de la búsqueda de información en bases de datos especializada en ciencia de la información y la recuperación respectiva de artículos, principalmente, se obtuvieron siete investigaciones publicadas en Journal of the American Society for Information Science and Technology, Ciência da Informação, Canadian Journal of Information and Library Science, ASLIB Proceedings: New Information y Journal of Information Science. Los artículos, y sus respectivos autores, se muestran en la tabla I.

\begin{tabular}{ll}
\hline Autor(es) & Título de artículo \\
\hline Mbambo, B., \& Cronjé, J. C. (2002). & $\begin{array}{l}\text { The internet as an information conduit in developing countries: An investigation of world } \\
\text { wide web usability among small and medium textile enterprises in Botswana }\end{array}$ \\
\hline $\begin{array}{ll}\text { Almeida Moraes, Giseli Diniz de, \& } \\
\text { Escrivão Filho, E. (2006). }\end{array}$ & \begin{tabular}{l} 
A gestão da informação diante das especificidades das pequenas empresas \\
\hline Bergeron, P. (1997).
\end{tabular} \\
\hline Verville, J., \& Halingten, A. (2003). & Information searches: A two-dimensional approach for ERP acquisition decision \\
\hline Chan, S. Y. (2001). & $\begin{array}{l}\text { The use of graphs as decision aids in relation to information overload and managerial } \\
\text { decision quality }\end{array}$ \\
\hline $\begin{array}{ll}\text { Choo, C. W., Bergeron, P., Detlor, } \\
\text { B., \& Heaton, L. (2008) }\end{array}$ & Information culture and information use: An exploratory study of three organizations \\
\hline De Saulles, M. (2007) & Information literacy amongst UK SMEs: An information policy gap \\
\hline
\end{tabular}

Tabla I. Relación de artículos referentes a la fenomenología de la información.

De acuerdo a la fecha de publicación, los artículos recuperados son de creación reciente, los años oscilan entre 1997 y 2008. Para el análisis de los resultados nos guiaremos por las categorías, previamente establecidas: 1) objetivos, metodología utilizada, tipo y tamaño de la organización, y el país o países donde se efectuaron los estudios; y, 2) identificación, tipificación y uso de la información en las organizaciones. En primer lugar, respecto a los objetivos de los artículos recuperados, se puede afirmar que atienden tanto el estudio del uso de la Internet (Mbambo \& Cronjé, 2002) como la administración de información (Almeida Moraes, Giseli 
Diniz \& Escrivão Filho, 2006; Bergeron, 1997), la sobrecarga de información (Chan, 2001), la cultura informativa y el uso de la información (Choo, Bergeron, Detlor \& Heaton, 2008), y la alfabetización informacional (De Saulles, 2007), principalmente. En el siguiente nivel de análisis, en cuanto a la metodología utilizada, predominaron los estudios descriptivos con uso de metodología cualitativa y el apoyo de instrumentos como el uso de la entrevista y el cuestionario (tabla II).

\begin{tabular}{ll}
\hline Autor(es) & Metodología utilizada \\
\hline Mbambo, B., \& Cronjé, J. C. (2002). & $\begin{array}{l}\text { Análisis de contenido, estudio de caso. Procedimiento: análisis en tres listas de } \\
\text { discusión (internet y desarrollo); test de usabilidad de la WWW. }\end{array}$ \\
\hline $\begin{array}{l}\text { Almeida Moraes, Giseli Diniz de, \& } \\
\text { Escrivão Filho, E. (2006). }\end{array}$ & $\begin{array}{l}\text { Investigación descriptiva-exploratoria; uso de } \\
\text { muestreo; instrumentos: cuestionario, entrevista personal, observación directa; } \\
\text { procedimiento, investigación documental, análisis documental, análisis de } \\
\text { resultados }\end{array}$ \\
\hline Bergeron, P. (1997). & $\begin{array}{l}\text { Metodología cualitativa; instrumento: entrevista abierta; muestreo (tamaño de la } \\
\text { organización, actividad y zona geográfica). }\end{array}$ \\
\hline Verville, J., \& Halingten, A. (2003). & Estudio de caso múltiple; colección de datos: entrevista semiestructurada. \\
\hline Chan, S. Y. (2001). & Tipo de estudio experimental \\
\hline Choo, C. W., Choo, C. W., Bergeron, & $\begin{array}{l}\text { Estudio exploratorio en tres organizaciones de conocimiento intensivo, } \\
\text { aplicación de cuestionario en WWW }\end{array}$ \\
\hline De, Detlor, B., \& Heaton, L. (2008). & $\begin{array}{l}\text { Uso de información primaria (Reino Unido) y secundaria (Estados Unidos de } \\
\text { América) y su posterior examen y análisis; instrumento, cuestionario en línea } \\
\text { (Reino Unido) }\end{array}$ \\
\hline
\end{tabular}

Tabla II. Relación de artículos investigados y su metodología.

Continuando con la descripción de los hallazgos, de acuerdo al tipo y tamaño de las organizaciones analizadas se obtuvieron los siguientes resultados. Por otro lado, acerca del tipo de organización manufacturera estudiada, hay un predominio mixto en aplicación de la investigación, es decir, los estudios no sólo se aplicaron a un tipo de organización sino a varios (por ejemplo, servicios e industria); solamente hubo dos investigaciones que se refirieron a un tipo: a) textil (Mbambo y Cronjé, 2002); y, b) metalurgia (Almeida Moraes, Giseli Diniz y Escrivão Filho, 2006). De igual manera que en el tipo de organizaciones, cuando se revisó el tamaño, los investigadores las estudiaron en conjunto, por ejemplo: micro, pequeña y mediana (Mbambo y Cronjé, 2002); pequeña y mediana (De Saulles, 2007); y, pequeña, mediana y grande (Choo, Bergeron, Detlor y Heaton, 2008). Menos de la mitad de documentos encontrados seleccionaron solo un tamaño: pequeña (Almeida Moraes, Giseli Diniz y Escrivão, 2006) y grande (Bergeron, 1997; Verville y Halingten, 2003; uno de los estudios no menciona el tamaño de la organización estudiada (Chan, 2001).

Respecto al análisis por zona geográfica de las investigaciones, se puede afirmar que predominó el continente americano con cinco países y en segundo plano se ubicaron Asia, África y Europa con un país cada uno (Tabla III). Esto indica, en otras palabras, que más de la mitad de los países estudiados pertenecen al Grupo de los ocho, es decir, las naciones más industrializadas del mundo. Los países a los cuales pertenecen los sujetos de estudio de las investigaciones analizadas son los siguientes: Brasil (Almeida Moraes, Giseli Diniz y Escrivão Filho, 2006), Canadá, (Bergeron, 1997; Choo, Bergeron, Detlor y Heaton, 2008) Estados Unidos de América (Verville y Halingten, 2003), China (Chan, 2001) y Reino Unido (De Saulles, 2007). Es importante destacar que la investigación de De Saulles (2007) se realizó mediante el cotejo y comparación de información de dos países, Reino Unido y Estados Unidos de América.

Para la identificación de los recursos de información en las organizaciones manufactureras nos hemos remitido a las tipologías de análisis documental de las Reglas de Catalogación Angloamericanas. De las tipologías de estas Reglas, en las investigaciones estudiadas, se mencionaron: las monografías, los archivos de computadora y recursos digitales (3), y las personas. Esta última categoría ha sido añadida, dada la reincidente mención en las investigaciones estudiadas. 


\begin{tabular}{|c|c|c|c|}
\hline Autor(es) & Tipo de organización & Tamaño & País \\
\hline $\begin{array}{l}\text { Mbambo, B., \& Cronjé, } \\
\text { J. C. (2002). }\end{array}$ & Textil & $\begin{array}{l}\text { Micro, pequeña } \\
\text { y mediana }\end{array}$ & Botswana \\
\hline $\begin{array}{l}\text { Almeida Moraes, Giseli } \\
\text { Diniz de, \& Escrivão } \\
\text { Filho, E. (2006). }\end{array}$ & Metalurgia (4 organizaciones) & Pequeña & $\begin{array}{l}\text { Brasil (Sao Jose do Rio } \\
\text { Prato, Estado de São } \\
\text { Paulo) }\end{array}$ \\
\hline Bergeron, P. (1997). & $\begin{array}{l}\text { Banco (1); servicios financieros } \\
\text { (2); alimentos y productos afines } \\
\text { (1); productos de papel y celulosa } \\
\text { (1); textil (1); equipo de transporte } \\
\text { (1) }\end{array}$ & Grande & Canadá (Montreal) \\
\hline $\begin{array}{l}\text { Verville, J., \& } \\
\text { Halingten, A. (2003). }\end{array}$ & $\begin{array}{l}\text { Servicio de transporte (1); } \\
\text { Empresa de mantenimiento (1); } \\
\text { Empresa de mantenimiento (1); } \\
\text { Empresa de manufactura de } \\
\text { muebles (1) }\end{array}$ & Grande & $\begin{array}{l}\text { Estados Unidos de } \\
\text { América }\end{array}$ \\
\hline Chan, S. Y. (2001). & $\begin{array}{l}\text { Experimental, selección de } 40 \\
\text { administradores (mando medio) } \\
\text { voluntarios de tiempo completo, } \\
\text { con } 6.5 \text { años de experiencia }\end{array}$ & No se menciona & China (Hong Kong) \\
\hline $\begin{array}{l}\text { Choo, C. W., Choo, C. } \\
\text { W., Bergeron, P., } \\
\text { Detlor, B., \& Heaton, L. } \\
\text { (2008). }\end{array}$ & $\begin{array}{l}\text { Despacho legal (1), investigación } \\
\text { en saludo (1), ingeniería en } \\
\text { aviación (1). }\end{array}$ & $\begin{array}{l}\text { Pequeña, } \\
\text { mediana y } \\
\text { grande }\end{array}$ & Canadá \\
\hline De Saulles, M. (2007). & $\begin{array}{l}\text { Principalmente servicios (Reino } \\
\text { unido); servicios financieros, } \\
\text { gobierno, manufactura y salud. }\end{array}$ & $\begin{array}{l}\text { Pequeña y } \\
\text { mediana }\end{array}$ & $\begin{array}{l}\text { Reino Unido, Estados } \\
\text { Unidos de América }\end{array}$ \\
\hline
\end{tabular}

Tabla III. Tipo, tamaño y localidad de las investigaciones analizadas.

A continuación la explicación para cada categoría:

- Monografías. Cinco de siete investigaciones recurren a este tipo de información. Los registros administrativos fueron los más mencionados (reportes, relatorías, políticas), así como también otros documentos (gráficas, tablas, memoranda). En una investigación se mencionó el uso de magazines y periódicos (Verville y Halingten, 2003). Si hay que hacer un recuento de las organizaciones manufactureras que utilizan la categoría monografías, según su tamaño, se afirmaría que son las pequeñas, medianas y grandes; sobresaliendo éstas últimas (Bergeron, 1997; Verville y Halingten, 2003; Choo, Bergeron, Detlor y Heaton, 2008). En relación al tipo de sector al cual pertenecen las organizaciones manufactureras que usan las monografías son: metalurgia, productos de papel, textil y muebles.

- Archivos de computadora y recursos digitales. Seis de siete investigaciones mencionaron hacer uso de éstos. Los recursos Internet, la World Wide Web (WWW) y archivos de textos son los recursos utilizados por las organizaciones manufactureras. La investigación de Chan (2001) no define si las tablas y gráficas son en formato digital, por este motivo no se incluyen en la tabulación de este estudio.

- Personas como recurso de información. A excepción de la investigación de Mbambo y Cronjé (2002), los seis estudios restantes mencionan este medio de información en las organizaciones estudiadas, de los cuales destacan: los consultores externos, el personal dedicado a las ventas, las conferencias y seminarios, y las asociaciones profesionales, comerciales y de negocios.

\section{Método}

Estudio descriptivo de investigaciones empíricas en el cual se establecieron dos tipos de categorías para el análisis de los resultados: 1) objetivos, metodología utilizada, tipo y tamaño de la organización, y el país o países donde se efectuaron los estudios; y, 2) Identificación, tipificación y uso de la información en las organizaciones. El procedimiento de la investigación fue el siguiente: búsqueda de información documental en bases de datos especializadas en ciencia de la información (LISA, LISTA) en los cuales la configuración de la de búsqueda fue por palabra clave o tema y las palabras fueron: organization, information, industry ó manufacture. Estos términos en los idiomas español, francés y portugués fueron utilizados para la búsqueda en las 
mismas bases de datos y otras fuentes documentales, (catálogos de instituciones de educación superior e investigación). Posteriormente se realizó la recuperación, el análisis de los documentos y la presentación de resultados.

\section{Discusión}

En primer lugar, de acuerdo a las definiciones teóricas y los resultados de las investigaciones analizadas, se puede afirmar que hay elementos suficientes para encontrar que la ciencia de la información, mediante la Al y la ARI, bien puede apoyar los estudios de identificación, tipificación y uso de la información en las organizaciones manufactureras. Sin embargo, para la tipificación de los recursos de información encontrados ha sido insuficiente la lista proporcionada en las Reglas de Catalogación Angloamericanas, herramienta básica con la cual los profesionales de la información realizan el análisis documental en organizaciones de uso masivo de información (bibliotecas, archivos, museos, y centros de información y documentación especializados), puesto que recurrentemente se encontró que las personas pueden ser fuente y herramienta de información para la consumación de los objetivos organizacionales, específicamente en el desempeño de actividades de procesos de planeación; se tuvo que añadir el tipo: personas, para complementar dicha tipología. Ante este hecho, encontrar a las personas como un elemento más que puede producir, procesar y otorgar información, se cumple el vaticinio de Drucker (2003, p. 49) cuando afirmaba que la información sería uno de los activos dentro y fuera de las organizaciones; e incluso erigirse como trabajadores del conocimiento. Esta afirmación coincide y se amplía con lo que Liao y Tang (2004) sustentan al mencionar que la integración de la información afecta a la creación de capital humano mediante el conocimiento adquirido por los integrantes de una organización.

Las organizaciones, en general, como entidades que pueden producir, organizar, procesar, difundir y utilizar la información -incluyendo las personas- han encontrado una manera de bajar los costos en la manera de difundir y comunicar esa información mediante las tecnologías de la información (Drucker, 2003, p. 219), situación que puede poner en ventaja o desventaja a quienes las utilizan. Ventaja, si es que se tiene una clara idea de que son una herramienta cuyo conocimiento puede ser bien explotado; desventaja, pues, si no es posible contar con las habilidades y destrezas para su uso, se puede caer en el analfabetismo informacional.
Por otro lado, en general, la metodología cualitativa para el estudio de identificación, tipificación y uso de la información en las organizaciones, es factible en la medida en que encontramos evidencia de la utilización de estas.

Sin embargo, se observa que en la mayoría de los documentos analizados no hay criterios homogéneos para determinar la generalización de resultados, es decir, se mezclan sujetos de investigación, especialmente: a) por tipo de organización, servicios bancarios y organizaciones manufactureras, servicios de mantenimiento con fábricas, o despachos legales con ingeniería de aviación, instituciones gubernamentales con la industria, por mencionar algunos; y b) por el tamaño de la organización, pequeña y mediana, o en su defecto, pequeña mediana y grande.

La falta de establecimiento de criterios de homogeneidad en la investigación con metodología cualitativa demerita la confiabilidad de los resultados, afectando de esta manera a la consolidación de una ciencia de la información sólida en este campo. Al observar la selección del tipo y tamaño de las organizaciones, por parte de los autores, es necesario señalar que si se desea consolidar a la ciencia de la información como disciplina sólida habrá que trabajar bastante en la selección de criterios metodológicos. Es decir, deberán cuidarse los criterios de homogeneidad de los sujetos de estudio. Si existieran juicios o discernimientos heterogéneos sería conveniente consignarlo en los alcances y limitaciones de la investigación.

\section{Posibilidades de investigación futuras}

Ante la premisa de contar con escasas investigaciones que atiendan un tipo o tamaño de organización manufacturera se abre la posibilidad de proseguir este tipo de estudios, es decir, de identificar y tipificar los recursos de información, así como la manera en que se comparten, tanto al interior como en el exterior de las organizaciones manufactureras.

Para futuras investigaciones, a continuación, se presentan algunas propuestas y preguntas clave. Dentro de la estructura de las organizaciones manufactureras, ¿dónde y quienes generan y utilizan los recursos de información? ¿de qué manera se fluyen y comparten esos recursos de información? De acuerdo a los procesos administrativos de las organizaciones manufactureras, ¿en cuáles de éstos se concentra o se observa mayor o menor dinamismo en los flujos de información?

Posteriormente al uso de los recursos de información en las organizaciones manufactureras, 
¿es posible observar la generación de nuevos recursos de información, cuáles son y quienes los producen?

Es importante destacar que esta investigación se realizó mediante el enfoque de la administración de información, y teniendo el antecedente de que los recursos de información pueden generar conocimiento, ¿es posible estudiar los flujos de información a partir de la disciplina de la administración del conocimiento? En un contexto de los estudios multidisciplinarios, ¿es posible investigar los flujos de información en las organizaciones manufactureras a partir de la disciplina administrativa y los estudios organizacionales?, ¿cómo definen y valoran a los recursos de información dichas disciplinas?

\section{Conclusiones}

En definitiva, hay pocas investigaciones encargadas de describir los recursos de información en las organizaciones estudiadas. Por tipo de industria, se encuentra la textil y la manufacturera, y por tamaño de las organizaciones sólo una: la pequeña. Los hallazgos, producto de la investigación, indican que las investigaciones estudiadas, en su mayoría, realizan una mezcla de sujetos de estudio, sin hacer énfasis en una sola organización o en un grupo de organizaciones manufactureras, es decir, no hay una buena elección de criterios de homogeneidad. Se puede concluir, además, que las investigaciones encontradas prefieren aplicar la metodología cualitativa.

Las preferencias de uso de información en las organizaciones manufactureras estudiadas se concentran en las monografías, en los archivos de computadora y los recursos digitales, y en las personas. Con estas aseveraciones, se puede decir que se han cumplido los objetivos de identificar y tipificar las fuentes de información.

La administración de recursos de información es, en la práctica, relativamente nueva. Sin embargo, su utilización en las organizaciones manufactureras es parcial: aún no se refleja en la aplicación de procesos completos de la administración —planeación, organización, dirección y control.

En casi la totalidad de los estudios analizados se menciona a las personas como fuente de información. Entonces, lo recomendable para los profesionales de la información es incluir a estos elementos como consumidores y generadores de información; e incluso como instrumentos para la toma de decisiones. Las personas se están valorando como capital intelectual de las organizaciones manufactureras.
Por último, los escasos estudios de los recursos de información, su tipificación y administración, en las organizaciones manufactureras, desde un enfoque de la ciencia de la información, abren la posibilidad de realizar investigaciones en este campo del conocimiento.

\section{Reconocimientos}

Un especial agradecimiento al Consejo Nacional de Ciencia y Tecnología (México) por el apoyo económico para desarrollar la tesis Estudio de los flujos de información en las organizaciones bajo tres enfoques: administrativo, organizacional y ciencias de la información, de la cual esta investigación forma parte. A la Universidad Nacional Autónoma de México y la Universidad de Zaragoza por los apoyos y facilidades otorgados para una estancia de investigación. A mis tutores Dr. Juan Voutssás Márquez, Dra. Georgina Araceli Torres Vargas y al Dr. Salvador Gorbea Portal. Al Dr. Francisco Javier García Marco por su hospitalidad y atinadas observaciones durante mi estancia de investigación en la Universidad de Zaragoza.

\section{Notas}

(1) En sus investigaciones, Oppenheim, Stenson y Wilson (2003a, 2003b, 2004) identifican los atributos de la información, y concluyen que a ésta se le puede considerar, en el ámbito de las organizaciones, como activo informativo.

(2) Se recomienda el artículo de Burk y Horton (1988, p. 4850 ), quienes a finales de la década de los ochenta, elaboraron dos listas ilustrativas de recursos de información, una de recursos de información interna y otra de los recursos de información externa.

(3) De las tipificaciones de información documental que no se mencionan en las investigaciones analizadas, pero que sí se categorizan en las Reglas de Catalogación Angloamericanas, son los siguientes: materiales cartográficos, gráficos para proyectar, gráficos no proyectados, micro formatos, grabaciones sonoras, arte impreso general, arte impreso específico, y películas y grabaciones.

\section{Referencias}

Almeida Moraes, Giseli Diniz de; Escrivão Filho, E. (2006). A gestão da informação diante das especificidades das pequenas empresas. // Ciência da informação. 35:3 (2006) 124-132.

Amaya de Heredia, M.; Joint Steering Commitee for Revision of AACR; American Library Association. (2004). Reglas de catalogación angloamericanas actualización de 2003. ( $2^{\mathrm{a}}$ ed. revisión de 2002). Bogotá: Rojas Eberhard.

Archaira, P.; Pani, A. (2008). Information management. // Sahu, Ashok K., ed. Information management in new millennium: opportunities and challenges for library professionals. New Delhi: Ess Ess, 2008.

Bergeron, P. (1997). A qualitative case study approach to examine information resources management. // Canadian Journal of Information and Library Science. 22:3/4 (1997) 1-19.

Burk, C. F.; Horton, J.; Forest W. (1988). InfoMap: A complete guide to discovering corporate information resources. Englewood Cliffs, N.J.: Prentice Hall, 1998. 
Chan, S. Y. (2001). The use of graphs as decision aids in relation to information overload and managerial decision quality. // Journal of Information Science. 27:6 (2001) 417-425.

Choo, C. W.; Bergeron, P.; Detlor, B.; Heaton, L. (2008). Information culture and information use: An exploratory study of three organizations. // Journal of the American Society for Information Science and Technology. 59:5 (2008) 792-804.

De Saulles, M. (2007). Information literacy amongst UK SMEs: An information policy gap. // Aslib Proceedings: New Information Perspectives. 59:1 (2007) 68-79.

Drucker, Peter F. (2003). La empresa en la sociedad que viene: los seis factores que están transformando al mundo que conocemos. Argentina: Empresa active, 2003.

Herrero, R.; Torres, M. (1993). Source of spanish economic information on domestic industry. // Kalseth, K.; Portela Peñas, P.; Stanton, T. M. (eds.) (1993). Information management for business: Proceedings of the FID information for industry committee seminar on information management for business, held in Madrid, Spain on 26 october 1992. La Haya: International Federation for Information and Documentation, 1993. 39-83.

Horton, J.; Forest, W. (1993). What does information resources management have to do with business? // Kalseth, K.; Portela Peñas, P.; Stanton, T. M. (Eds.) (1993). Information management for business: Proceedings of the FID information for industry committee seminar on information management for business, held in Madrid, Spain on 26 october 1992. La Haya: International Federation for Information and Documentation, 1993. 1-15.
Kirk, J. (1999). Information in organizations: directions for information management. // Information Research, 4:3 (1999). http://informationr.net/ir/4-3/paper57.html (201006-14).

Liao, S. S.; He, J. W.; Tang, T. H. (2004). A framework for context information management. // Journal of Information Science. 30:6 (2004) 528-539.

Martínez Musiño C. (2010). El valor de la información, su administración y alcance en las organizaciones. // Revista mexicana de ciencias de la información. 1:2 (2010) 10-20.

Mbambo, B.; Cronjé, J. C. (2002). The internet as an information conduit in developing countries: An investigation of World Wide Web usability among small and medium textile enterprises in Botswana. // ASLIB Proceedings: New Information. 54:4 (2002) 251-259.

Oppenheim, C.; Stenson, J.; Wilson, R. M. S. (2003a). Studies on information as an asset I: Definitions. // Journal of Information Science. 29:3 (2003a) 159-166.

Oppenheim, C.; Stenson, J.; Wilson, R. M. S. (2003b). Studies on information as an asset II: Repertory grid. // Journal of Information Science. 29:5 (2003b) 419-432.

Oppenheim, C.; Stenson, J.; Wilson, R. M. S. (2004). Studies on information as an asset III: View of information professional. // Journal of Information Science. 30:2 (2004) 181-190.

Orna, Elizabeth. (1990). Practical information policies: how to manage information flow in organizations. England: Gower, 1990.

Verville, J.; Halingten, A. (2003). Information searches: A two-dimensional approach for ERP acquisition decision. // Journal of Information Science. 29:3 (2003) 203-209. 